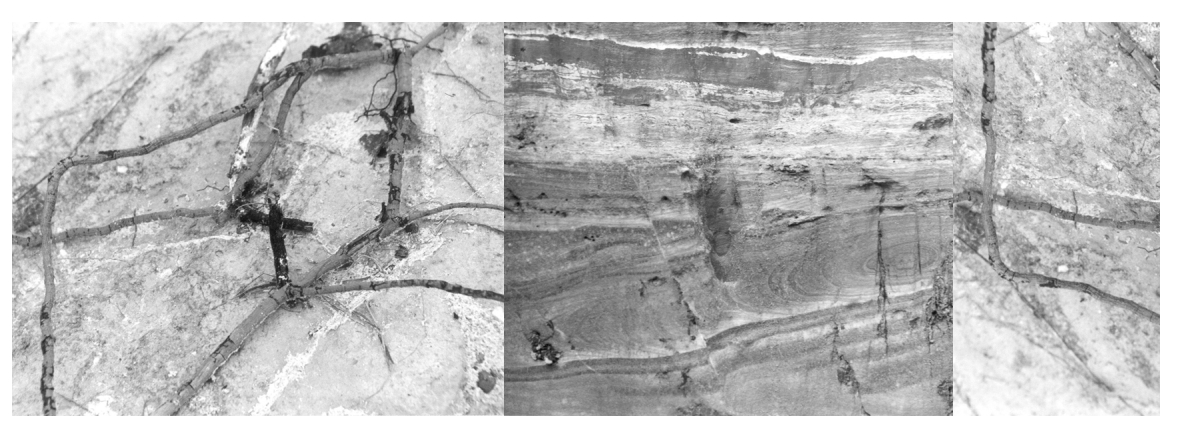

\title{
Los caminos de la Universidad en un contexto de cambio social
}

Araceli Estebaranz García ${ }^{1}$

GARCÍA, A. E. The course of the university in a period of social change, Interface - Comunic, Saúde, Educ, V.7, n.13, p.9-26, 2003.

In this article, we reflect on the current problems and paradoxes of Higher Education. Also, we present the prospects for improvement in the immediate future. The functions that ought to be part of Higher Education in today's society are examined, as are the quality demands of a service that increasingly affects a larger number of citizens, and on which social change to a large degree depends. Finally, suggestions concerning teaching methodologies.

KEY WORDS: Higher education; social change; teaching methodology.

En este artículo se hace una reflexión sobre la problemática actual y las paradojas de la Enseñanza Superior. También se ofrecen perspectivas de mejora para el futuro inmediato. Se analizan las funciones que debe cumplir la Educación Superior en la sociedad de hoy, así como las exigencias de calidad de una formación que afecta cada vez a un mayor número de ciudadanos, y del cual depende en gran medida la transformación de la sociedad a niveles nacionales e internacionales. Más adelante se hacen propuestas didácticas para la innovación en la formación que proporciona.

PALABRAS CLAVE: Educación Superior; cambio social; pedagogía universitaria.

${ }^{1}$ Catedrática, Departamento de Didáctica y Organización Escolar, Faculdad de Ciencias de la Educación, Universidad de Sevilla. <tebaranz@us.es> 


\begin{abstract}
La Universidad hoy debe servir para formar una amplísima masa crítica de ciudadanos exigentes y capaces de deliberación política circunstanciada, y de miembros activos $y$ participantes en empresas económicas $y$ asociaciones de toda índole que prosperen en un medio institucional exigente.
\end{abstract}

(Pérez, 1995)

\section{Las funciones de la Universidad en el nuevo siglo}

Voy a comenzar con un breve repaso de las tres grandes etapas que se pueden reconocer en la historia de la Universidad. Alvin Toffler (1996), en su obra La Tercera Ola, reconocía una diferencia de foco y de funciones: Lo que él llama la primera ola, ha sido una época larga, en la que a la Universidad se le encomendó la investigación y difusión de la Ciencia. Era la Universidad de elites. Una institución para minorías, bien dotadas y capacitadas para crear y utilizar, o proponer la utilización de la ciencia. Claramente se le encomendaba la formación de los futuros dirigentes intelectuales de la sociedad.

El crecimiento económico, la exigencia de una mayor calificación para una mayor diversidad de puestos de trabajo, y la conquista de la democracia (en España, en la segunda mitad del siglo XX) provoca un cambio en la concepción y en la realidad de la Universidad. La segunda ola se caracteriza por el interés de dar oportunidades a la mayor cantidad de ciudadanos posible de acceder a la cultura, de disfrutar de los más altos grados de educación. Así se llega a conquistar la Universidad de masas, como Institución que potencia la igualdad social. Pero este crecimiento no ha ido acompañado de medidas adecuadas para dar respuesta a esas exigencias de la sociedad, y hoy nos encontramos inmersos en el discurso de la calidad. La cuestión hoy es cómo lograr una Educación Superior de calidad para el mayor número de ciudadanos posible. $Y$ esto supone ir construyendo caminos. La calidad es un camino por hacer. Parafraseando al poeta A. Machado "Caminante no hay camino, sino estelas en la mar". Y en el mar se pueden trazar todos los caminos que se imaginen, con brújula, o con GPS, que proporcionan orientación. Pero la técnica, que nos sirve para recorrer caminos, no nos los puede imponer.

Estamos en la tercera ola de la Universidad (como parte de la tercera ola que estamos viviendo en lo social, cultural, político, económico, tecnológico, científico e ideológico) y, como dice Toffler, "es una gran marea que levanta el mundo, lo asentado, y que está creando un entorno nuevo en el que trabajar, jugar, casarse, criar hijos y jubilarse" (p.10). Es una tercera ola levantada por la Globalización y por el impacto de las Nuevas Tecnologías, que yo no voy a analizar desde el punto de vista económico y sus problemas, pero que indudablemente están generando nuevas relaciones, estilos de vida, modos de comunicación, nuevas formas de nacimiento y de vida, y una conciencia planetaria, pero, con ello también, nuevos problemas, nuevas necesidades y nuevas posibilidades de dar respuesta a problemas que son nuevos. Es decir, que hoy, a causa de la globalización, debemos ser conscientes de que nuestras acciones pueden influir decisivamente sobre el equilibrio ecológico planetario, y por lo tanto,

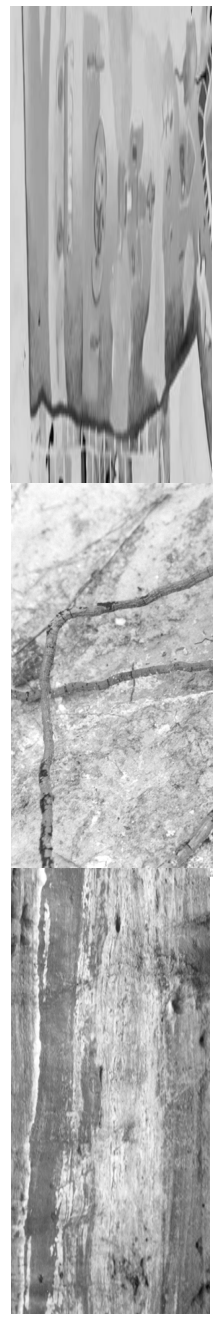


sobre la evolución de la sociedad global en su conjunto, y por ello hacen falta unos niveles mayores de formación y de anticipación, para que podamos encontrar antes las soluciones convenientes. Aunque el objetivo es claro. Al menos sabemos dónde queremos llegar: Mayores niveles de educación para todos los ciudadanos que lo deseen y hayan logrado un nivel mínimo de formación necesaria para acceder a estudios superiores. El camino de la equidad es irrenunciable. Pero lo importante no es la cantidad, meta a la que ya sabemos cómo llegar, sino que lo importante es la calidad.

¿Cómo entender la calidad de la Universidad? Nuestro trabajo nace de un conocimiento, comprensión y análisis de los problemas, características, e idiosincrasia de nuestra sociedad cercana, y la propuesta que hacemos está marcada por una especificidad concreta, pero con bastantes relaciones con el contexto general de la enseñanza en España, y de la Universidad española y europea, incluso, en el momento actual, en el que se percibe claramente una necesidad de Reforma profunda, que afecta a la misma identidad de los Estudios Superiores. Y que analizando los textos de la Unesco sobre el tema, vemos que en alguna medida en el ámbito de principios pueden generalizarse, porque se entiende que la calidad del sistema de Educación Superior debe evaluarse según la correspondencia entre lo que la sociedad espere de las instituciones y lo que ellas hacen. Ello requiere visión ética, imparcialidad política, capacidad crítica, $y$, al mismo tiempo, una mejor articulación con los problemas de la sociedad y del mundo del trabajo, basando las orientaciones a largo plazo en las necesidades y finalidades de la sociedad, incluyendo el respeto a la cultura y la protección ambiental (Unesco, 1998b).

Un acercamiento didáctico a la calidad tiene que ver con las funciones que se encomiendan a la Universidad; funciones derivadas de las nuevas necesidades sociales:

La custodia y promoción de la cultura. La Universidad es una institución cultural. Una institución para la vivencia cultural. El "conservatorio vivo del patrimonio de la Humanidad" (Delors, 1996, p.153), donde el debate sobre los grandes problemas éticos y científicos actuales cobra todo su sentido (Benedito, 1998), y además es posible por la autonomía que posee, frente a los poderes políticos, económicos, o de otra índole. La Universidad, en primer lugar, debe proporcionar una cultura que se reconoce por un interés por conocer, $y$ un peculiar modo de pensar, normalmente opuesto al utilitarismo, que se enriquece con el debate y el análisis crítico.

Desarrollar el saber $y$ difundir el conocimiento, aumentando la base de conocimiento de la sociedad, a través de la investigación básica y aplicada. Es una institución obligada a la investigación, sensible a los problemas de la sociedad para producir el conocimiento que necesita con el fin de progresar hacia mayores niveles de calidad de vida para todos. El conocimiento es un medio de cohesión social, y de profundización en la democracia (Michavila $\mathcal{E}$ Calvo, 1998). La Universidad tiene sentido como institución cuyas actividades se destinan al enriquecimiento intelectual, moral y material de la sociedad (Bricall, 2000). Por ello, la necesidad de apertura y permeabilidad a la sociedad.

Proporcionar formación profesional a sus alumnos, y facilitar el desarrollo profesional de sus propios profesores $y$ de otros profesionales. Es 
una institución formativa. $Y$ esta formación es necesaria como respuesta a las necesidades sociales y profesionales de la sociedad. Por ello, la Universidad tiene que transformarse, ofrecer no los saberes de siempre, sino los saberes que responden a preguntas.

Contribuir a la equidad de la sociedad por la educación de un mayor número de ciudadanos, por más canales, $y$ con diversidad de niveles de formación, y por más tiempo. Debe ser un asunto de Estado. En la sociedad de la información hay que procurar que el conocimiento sea un bien distribuido al alcance de todos. Así se concibe la Universidad como una agencia de educación permanente. Ello ha exigido la diversificación de los estudios de Tercer Ciclo, integrando además de los programas de Doctorado los cursos de Especialización, de Experto y de Master.

Proporcionar la oportunidad de desarrollo personal, y como tal, tiene una función de revelación -descubrimiento y desarrollo - de las capacidades individuales. Es una institución de aprendizaje en todos los ámbitos del desarrollo personal. Ello supone que debe buscar los métodos de enseñanza que promuevan el desarrollo de la capacidad de aprendizaje y de construir conocimiento.

La cuestión es que puestas en fila las funciones se ven claras. Si se unen en una sola proposición ilusionan: "La universidad debe seguir siendo el templo de la cultura, de la ciencia, de la reflexión intelectual, de la formación humana en su plenitud" (Benedito, 1998, p.51). Conjugar las exigencias de todas ellas es complicado, difícil y costoso. Conjugar cantidad y calidad es un reto. ¿Quién debe asumir el reto?. Es una preocupación general, $y$ debe ser una responsabilidad compartida. Pero hay que tener en cuenta que hay muchas realidades universitarias. Hay diferencia de medios, de recursos humanos (porcentaje de doctores, por ejemplo), de experiencia y conocimiento, de posibilidades de investigación etc.

En la encrucijada de caminos: las paradojas de la enseñanza superior

Masificación progresiva en la enseñanza superior, pero a la vez, reducción relativa de los recursos económicos materiales y humanos que se les asignan. Cuando estudiamos las relaciones entre Universidad y Sociedad, encontramos una gran demanda del servicio que no va acompañada de los necesarios apoyos del sistema (traducidos en apoyo económico) por parte de los Órganos e instrumentos de vinculación entre Universidad y Sociedad (Consejo Social, particularmente), para todos los objetivos que se encomiendan a la Institución universitaria. Por ello, en nuestro contexto se ve la necesidad de un gran pacto social y político sobre el modelo de financiación.
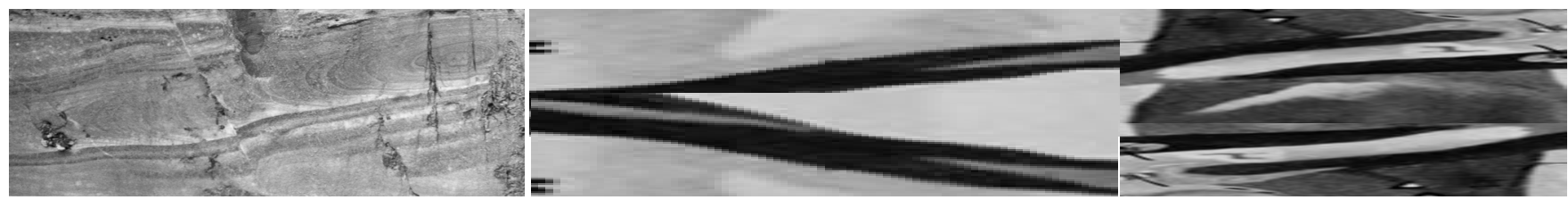
Adaptación a nuevas modalidades de empleo pero sin coste, o con el mínimo. Es el caso de nuestra Reforma Curricular realizada fundamentalmente durante los años 1990 y 1991, por la que se crean 133 titulaciones específicas, pero para la implantación de nuevas titulaciones se aprueban fundamentalmente aquellos proyectos de las Universidades que supongan coste cero. Ello quiere decir que lo nuevo tiene que enseñarse con los medios y recursos humanos de que disponemos.

Masificación y a la vez mecanismos de exclusión. Las mujeres, que son más en cuanto al número de matricula y de egresados, siguen estando poco representadas en el campo científico y técnico y en el de gestión de la Universidad. Y si imprimen su carácter de más apertura, consenso y acogida, se encuentran con problemas, y a veces con la exclusión.

Necesidad de internacionalización frente a la contextualización de la tarea, por otra parte. Así tenemos muy claro que la investigación debe promover la innovación, interdisciplinariedad y transnacionalidad de los proyectos (Unesco, 1998b, Art.7). Pero a la vez se insiste en la importancia del papel que las Universidades locales y nacionales pueden desempeñar en el desarrollo de su país (Delors, 1996). Investigaciones contextualizadas y efectivas, y más donde son más necesarias.

Pero la Universidad ya no tiene el monopolio de la investigación. Cada vez más las multinacionales tienen sus propios centros de investigación y formación, y existen cada vez más PYMES especializadas en la investigación y en el desarrollo. Ello supone la necesaria colaboración entre ambas. Ahí surgen los parques científicos y tecnológicos, en los que pueden colaborar las empresas, las universidades, las administraciones regionales o locales. Esto parece una tendencia en alza en los próximos años. Pequeñas sociedades mixtas de investigación y desarrollo, y de formación. Unesco pronostica que en adelante las Universidades podrán ser también accionistas. Esto supone un aumento de la tercera vía de financiación. Ahora estamos en la idea de colaboración por contratos para proyectos con las administraciones públicas o las empresas. Lo cual plantea problemas de tiempo y de recursos, sobre todo humanos. En adelante es preciso la planificación estratégica. Si la Universidad debe investigar para dar respuesta a las necesidades laborales, sociales y morales de la sociedad, hay que invertir en capital humano. No se puede dedicar demasiado tiempo a la enseñanza, a la enseñanza de masas, por parte de jóvenes investigadores, en los mejores años de su producción y creación, sin que se resienta la investigación y la calidad de la vida humana.

Nuevas Tecnologías de la Información y la Comunicación - la mayor parte tienen su origen en la investigación científica fundamental llevada a cabo en las Universidades y desarrolladas en ellas o en colaboración con empresas. Pero en la práctica siguen siendo aún poco utilizadas en la educación, o en la formación, y principalmente como complementarias del ambiente de aprendizaje real, y sobre todo en los estudios relativos al Tercer Ciclo. Actualmente se empiezan a impartir asignaturas por el sistema de Teleformación.

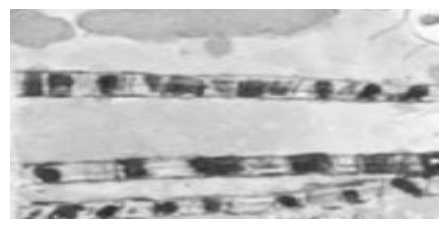


La apertura y la flexibilización de la formación son necesarias pero sigue habiendo una gestión rígida del tiempo del docente a corto plazo. En la sociedad de la información, el paradigma de la enseñanza debe cambiar por el del aprendizaje. El docente pasa a ser autor y administrador del aprendizaje. Pero en la Universidad sigue habiendo demasiada enseñanza y quizá poco aprendizaje. $Y$ en este sentido, sabemos que las Nuevas Tecnologías aportan flexibilidad al aprendizaje y a las tareas de investigación. Por ello, se deberá generalizar en la mayor medida posible la utilización de las Nuevas Tecnologías para reforzar el desarrollo académico, ampliar el acceso, lograr una difusión universal y extender el saber, y

facilitar la educación durante toda la vida.

Formación igeneral o especializada? Cuando se critica a la Universidad se le pide formación especializada. Pero la Universidad, y sobre todo la de masas, debe proporcionar formación general, plurivalente, aún dentro de las carreras, que ya suponen una cierta especialización. En ese sentido, muchos científicos o especialistas en el campo de las ingenierías y tecnologías reconocen el valor de la formación general, para posteriormente especializarse. Por otra parte, datos de la Unesco indican que el

conocimiento queda obsoleto en poco tiempo. Ello abogaría por la necesidad de la formación más general.

Pero, además, la Universidad debe proporcionar una mejor orientación a los estudiantes respecto a su futuro laboral, ello supone que es adecuado conservar el carácter pluridimensional de la enseñanza superior, porque da más versatilidad a los titulados para adaptarse al mercado laboral, y para orientarse sobre si trabajar en el mercado, en la industria, en la educación o en la investigación. $Y$ precisamente el tercer ciclo de los estudios

universitarios supone la especialización: o para la investigación o para las funciones de responsabilidad en el mercado de trabajo. Una colaboración entre ambos mundos puede y debe ser útil a través de contratos, programas en Facultades y Departamentos, que pueden competir con otras universidades, y determinar el nivel logrado en determinados parámetros de calidad, lo que redundará en la financiación.

Los caminos hacia la calidad: la necesaria reconstrucción de la Universidad

La situación actual de la Universidad puede considerarse como un proceso de reconstrucción de su identidad para poder adaptarse y transformarse según las necesidades de la sociedad actual (Benedito, 1998). Pero paralelamente la búsqueda de la identidad lleva consigo la necesidad de calidad y de prestigio. Así, el futuro de la Universidad debe resolver algunos retos, como el incremento de la calidad de la enseñanza aún con las dimensiones que tiene la Universidad actual y la futura; la prestación eficiente y eficaz del servicio público de Educación Superior a la sociedad, atención a las demandas de formación de un número creciente de personas de la tercera edad, la búsqueda del equilibrio entre la investigación básica y la aplicada, y recuperar su papel orientador, iluminador y provocador de los cambios sociales, económicos y culturales. Y para ello, necesita afrontar los cambios y las Reformas legislativas necesarias y graduales que hagan posible el logro de 


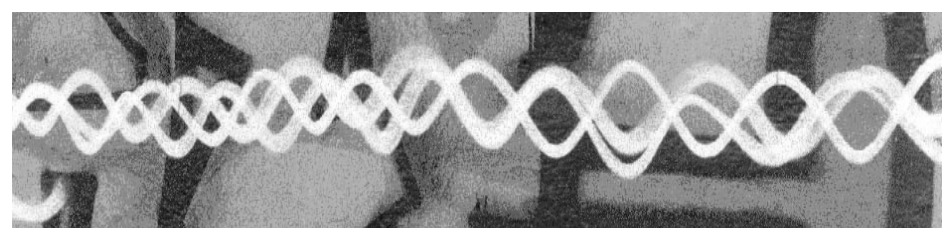

los objetivos señalados. Podemos señalar algunos principios para orientar el cambio:

Profundizar en la equidad. La educación superior seguirá siendo de masas; ha sido una conquista de la democracia, y a causa de la innovación y del progreso tecnológico, las economías exigirán cada vez más competencias profesionales, que requieran un nivel elevado de estudios. Por eso hay más demanda de estudios superiores. A nivel Internacional el Proyecto Marco de Acción Prioritaria para el Cambio y el Desarrollo de la Educación Superior, presentado en la Conferencia Mundial sobre la Educación Superior, celebrada en París del 5 al 9 de octubre de 1998, en su apartado 1.2 (Unesco, 1998a), recomienda a los Estados que se esfuercen por garantizar un nivel de educación superior adecuado a las necesidades actuales de los sectores público y privado de la sociedad..., sobre todo a aquellos países cuyo nivel medio de estudios superiores no alcanza los niveles aceptados internacionalmente.

La permeabilidad. Las relaciones entre la Universidad y el mundo social, cultural y laboral, deben ser cada vez más estrechas, porque ambas se beneficiarán. De hecho, se ve a la Universidad como una institución con fines de desarrollo regional. La relación es más apropiada con las realidades más cercanas. Por ello, se pide a las Instituciones de Educación Superior permeabilidad, que posibilite el conocimiento mutuo, la apertura al entorno (a través del diálogo por medio de los representantes sociales en el Consejo Social de la Universidad) y relaciones de colaboración. Se reconoce esta necesidad pero también la dificultad de que se materialice si no cambia la cultura universitaria, desde el polo de lo académico a la colaboración interdisciplinar $y$ a las relaciones entre los investigadores y los usuarios o beneficiarios de la investigación, así como la propia estructura de la Universidad.

Cambio en el empleo y cambio de la Universidad: tecnología, saber y empleo

La revolución de la información está favoreciendo la mutación de la vida social y del trabajo. La Educación Superior, como la de otros niveles, debe prepararse y preparar para la sociedad del saber, de la información y de la educación, o del aprendizaje. Probablemente la tarea más importante de cualquier sociedad o empresa. De tal manera que la Sociedad de la Información ha llegado a incorporar el concepto de "valor añadido" a las empresas de producción, y a las empresas de formación. El "valor añadido" de las empresas hoy se define en función del saber. Así como la sociedad industrial se orientó hacia el valor producto; la sociedad de la información valora el saber. Las Nuevas Tecnologías han llegado a revalorizar el saber, y el saber conduce a la formación como un valor añadido al mercado globalizado de hoy, de tal manera que las empresas tecnológicas han creado un puesto de trabajo, o incluso Departamento -según el tamaño de la 
empresa- dedicado a la Gestión del Conocimiento, como bien distribuido dentro de la propia empresa que es necesario organizar.

Hay una relación entre saber y empleo. De forma que en Europa, y España aún más, que tiene un mayor índice de paro (9\%) que el resto de los entornos, un planteamiento que cabe hacerse es "algo estamos haciendo mal en el saber, porque no estamos siendo competitivos en el hacer". De hecho, en el análisis de las tendencias de empleo se ve claramente que hay un alza del Sector cuaternario de producción: Las NTIC: robótica, informática, telemática, que van a influir en la modalidad de formación que es la teleformación.

En varias ocasiones se ha atribuido el problema de la falta de empleo a la tecnología. Kyriakou (1997), experto de la Comunidad Europea, economista del Instituto de Prospectiva Tecnológica y Científica de Sevilla, comentaba un reciente estudio estadounidense que avala su defensa de que el progreso tecnológico genera riqueza: las empresas que usan tecnologías avanzadas pagan salarios más elevados, ofrecen mejores empleos y más seguros, $e$ incrementan el empleo más rápidamente. En su opinión, en Europa hay más paro por el retraso en la incorporación de la Tecnología de la Información. Y aporta una idea interesante: "la vulnerabilidad de un trabajo depende de lo fácil que se pueda aprender por otros trabajadores o por máquinas". Entonces el problema del paro no reside en tareas especializadas o no especializadas sino que la diferencia está entre tareas repetitivas, susceptibles de la reproducción por ordenador y las tareas "irreducibles", netamente creativas. Así la artesanía no reducible y no informatizable, incluso las tareas propias de un trabajo manual (p.ej. la jardinería), pueden quedar a salvo de los efectos de las Nuevas Tecnologías. En este sentido, la formación, la educación es la clave del éxito para la sociedad, el trabajo y la economía. Promover valor añadido en una manera que sea difícil de imitar (por una máquina o por una persona) es la clave de la preservación del empleo y la cosecha de beneficios atractivos.

La Universidad y la evolución del mercado laboral. Es cierto que en el mundo de hoy disminuyen los obreros, o empleados del dueño de una empresa, y se multiplican las máquinas; ello supone que aumentan las tareas de supervisión, selección, marketing, formación, organización, y control de calidad de procesos; aumentan así las necesidades de cualificación (Delors, 1996). La Universidad es creadora de conocimientos y los recursos cognoscitivos tendrán cada día más importancia que los materiales como factores de desarrollo. Son las Universidades las que forman a los dirigentes intelectuales y políticos, a los jefes de empresa del futuro, a los generadores de empresas futuras, $y$ a una buena parte del cuerpo docente (Delors, 1996); aunque no sólo, también hay otras instituciones de Educación Superior.

La formación universitaria y el trabajo. La sociedad exige más nivel de formación, pero también critica a la Universidad como institución costosa y sin rendimientos: "fábrica de parados". Pero los datos indican que la Universidad es una "fábrica de ocupados"; los datos no ofrecen duda de que a mayor nivel educativo conseguido hay una mayor tasa de actividad y una 
menor tasa de desempleo, y además los salarios de los trabajadores con título universitario son más altos. Ello supone que sí hay correspondencia entre necesidades sociales y formación universitaria, y que son rentables los estudios universitarios tanto individualmente considerados como socialmente. El índice de paro de los universitarios de carreras de ciclo corto es menor que el del resto de la población y aún menor el de las carreras de ciclo largo. La estadística muestra que habiendo más universitarios ha subido el empleo de los mismos en los últimos 30 años. Sin embargo, hay un dato de interés: el autoempleo de las mujeres es menor. Por ello, hay empresas de formación empeñadas en orientar la actividad formativa a potenciar la capacidad de iniciativa de las mujeres: a formar mujeres emprendedoras.

¿A qué se debe el índice de mayor empleo de los universitarios? Parece que se debe precisamente a que éstos disponen de una mayor flexibilidad y habilidad para afrontar los cambios que tienen lugar en los sistemas culturales y productivos (Bricall, 2000).

Pero la necesaria permeabilidad de la Universidad a los cambios sociales exige adoptar medidas de respuesta, tales como las siguientes: innovación curricular - cambios en los Currículos, atendiendo a los principios de la diversificación curricular (ofrecer nuevas carreras y titulaciones en función de las demandas sociales y profesionales); definir los planes de estudio en función de la orientación profesional de cada titulación (el perfil profesional puede servir de guía para la selección de contenidos, la organización del tipo de prácticas más convenientes (Zabalza, 1999); la flexibilización de los currículos, en función de la propia flexibilidad del mercado laboral y de las profesiones, $y$ del reconocimiento de la capacidad de los estudiantes «dentro de unos límites imprescindibles» para elaborar su propio itinerario formativo; la actualización permanente de los Planes de Estudio (equilibrar una cierta estabilidad con el reajuste de los componentes más variables. $Y$ ello supone la posibilidad de cambiar o «redenominar» las disciplinas en función de los avances científicos en cada campo (Zabalza, 1999); asesoramiento al estudiante (medidas paralelas de ayuda o apoyo para que los estudiantes puedan hacer elecciones responsables y apropiadas a sus expectativas profesionales (Bricall, 2000); la organización colaborativa del Practicum con las empresas o entidades «colaboradoras» (Marcelo \& Estebaranz, 1998), para resolver el reto de la falta de formación práctica y de especialización de los graduados universitarios, de los que suele acusarlos la sociedad, en general, y que da lugar a una de las paradojas que vive la Universidad. Por ello, la primera idea a plantear es la necesidad de cooperación entre la Universidad y la empresa pública y privada.

Por otra parte, darle sentido al Practicum es una tarea cada vez más exigente. La colaboración con centros de empleo, centros de formación, empresas etc. exige contar con una red estable de centros, con los que se puede ir planificando $y$ darle sentido a cada tarea dentro de un currículum; $y$ profesores de prácticas estables, y enterados e interesados en esta formación, para que no sean únicamente unos créditos que se usan para cubrir el horario de los nuevos profesores, que pueden ser contratados incluso a mitad de curso, o simplemente de aquellos profesores que tienen 
menos carga docente, pero cuyas materias y cuya especialización no tiene ninguna relación con el campo de trabajo en el que sus alumnos deben aprender el sentido de una profesión.

$Y$ esto es una cuestión de cultura. De una cultura de la formación universitaria y de la dedicación y orientación del currículum y del trabajo de todos y de la organización; cultura que no existe en la Universidad. $Y$ que por ello, es una cuestión de crear condiciones, de planificación estratégica, y de tiempo. No de tiempo de espera para que sucedan las cosas, sino de tiempo de trabajo para conseguir las cosas que pensamos.

\section{La necesidad de planificación estratégica}

Ello empalma con la necesidad de planificación estratégica. La planificación estratégica supone un análisis de lo que la Universidad debe ofrecer y puede ofrecer con éxito. Dónde poner el foco y qué acciones emprender de manera sincronizada para cumplir su misión.

La Planificación estratégica se presenta como herramienta de gestión participativa, que hace posible integrar los cuatro niveles de gestión interna: Universidad, Centro, Departamento y Materia de Enseñanza, anticipándose al futuro y planificando la oferta académica y formativa en torno a prioridades consensuadas, buscando la eficacia y el logro del éxito en la misión de la Universidad, frente a una actuación precipitada como respuesta a demandas del entorno. $Y$ ello exige tener en cuenta el principio siguiente:

Gestión democrática: autonomía y participación, y transparencia. El derecho a la Autonomía de la Universidad, supone la posibilidad de «autodisposición de las Universidades frente a los poderes externos (el Estado, la Administración Pública, y otras entidades jurídicas y económicas) que pueden condicionarlas en el cumplimiento de sus funciones: enseñanza, investigación, y formación de profesionales.

Sin embargo, el cambio se puede propiciar a través de la participación en los distintos órganos responsables de la gestión y la docencia universitaria (Facultades y Departamentos), y del desarrollo de la investigación. La investigación es el alma de la docencia, y se lleva a cabo a través de los Grupos de Investigación.

La participación del alumnado en los órganos de gobierno es un elemento activador importante. Los alumnos son agentes de innovación, por lo que aportan, o por lo que exigen. Deben tener la capacidad de formar Asociaciones culturales, y para ello necesitan medios: espacios para reunirse, tiempos, presupuesto económico, que les llega a través de una partida del presupuesto del Órgano de Gobierno en el que participan.

Los estudiantes pueden participar en los órganos orientadores de la Universidad. En la Universidad de la experiencia, en actividades y servicios sociales: por ejemplo, residir con ancianos, con un beneficio mutuo. La labor de los alumnos al servicio de la comunidad (residir y convivir con ancianos) respondiendo a las necesidades de comunicación de este colectivo social, que es independiente pero está necesitado de relación y apoyo humanos, figuran entre los factores que pueden enriquecer la función cultural y social.

Son agentes de innovación curricular, siempre que se les permita pensar en qué currículum debe configurar un Plan de Estudios, o en la propia 
metodología didáctica, desde la que llegan a ser agentes, constructores de su propio aprendizaje.

La transparencia supone conocimiento y bien hacer. También participación y difusión del conocimiento. ¿Qué es necesario hacer?; ¿A quién se encomiendan determinadas responsabilidades y por qué?; ¿En qué es necesario invertir?; ¿En qué se invierte?; ¿Quién lo decide?; ¿Por qué se decide así?; ¿Cuál es su rentabilidad?; ¿Quién hizo estudios de necesidades o de mercado, de demanda, de posibles usuarios, o consumidores, que justifiquen esa oferta y la negación de otras? etc., son preguntas cuyas respuestas deben ser claramente comprendidas y compartidas por la Comunidad Universitaria.

Innovación en formación. Los cambios que se deben operar afectan a los objetivos y a los métodos. La rapidez de los cambios tecnológicos ha mostrado que la especialización de los trabajadores queda obsoleta en poco tiempo.

a) Objetivos - formación de conocimientos generales y de carácter interdisciplinar; capacidad de resolver problemas y tomar iniciativas; potenciar actitudes positivas hacia el trabajo, desde el punto de vista personal; valoración del trabajo en equipo y desarrollo de la capacidad de cooperación; incorporar a los individuos en la toma de decisiones de los procesos productivos; cultura general, que exige sobre todo el sector servicios, que ocupa un lugar preeminente en el empleo, $y$ apertura al conocimiento y uso de las posibilidades que ofrece el entorno humano; potenciar el enseñar a hacer, que ha sido descuidado, y para lo que la Universidad necesita a la sociedad, la empresa, las organizaciones de cualquier tipo.

b) La innovación didáctica: de la enseñanza al aprendizaje - todo ello supone que los métodos deben integrar las diversas formas de aprendizaje que potencien la motivación, la implicación en el propio aprendizaje, y la cooperación. Y no hay más remedio que incorporar lo que hemos aprendido de la Universidad a Distancia (Delors, 1996; Dondi, 1998): uso y dosificación juiciosa de las Nuevas Tecnologías; los contactos personales, pueden ensanchar las posibilidades de aprendizaje.

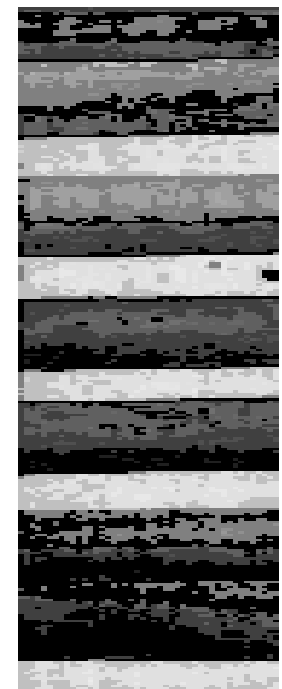

La formación debe ser el resultado de explorar cuestiones esenciales y de aprender a pensar sobre ellas de una manera disciplinada, no la consecuencia de dominar cincuenta o quinientos temas estipulados de antemano para cada curso. (Gardner, 2000, p.25)

Los procesos de enseñanza-aprendizaje son la esencia de la calidad de las instituciones formativas. El cambio necesario en la metodología en este nivel, como en otros, tiene que ver con las concepciones del aprendizaje y de la enseñanza y las estrategias metodológicas y ambientes necesarios para propiciar un aprendizaje individual y grupal, en cooperación y a través de la solución de problemas, que tienda hacia el autoaprendizaje y la investigación para adquirir y crear conocimiento de distintas fuentes y en la interacción con otros (Huber, 1998).

La calidad de los componentes estructurales. La Calidad de la educación supone calidad de los elementos: profesores, estudiantes, infraestructura y cultura. Así se ha visto en la Declaración Conjunta de los 
Ministerios de Educación de la Unión Europea de 1999 realizada en Bolonia; es un compromiso común de búsqueda de la calidad.

Selección y formación pedagógica de los docentes. El concepto de "valor añadido" como el saber disponible en una empresa para afrontar los problemas de producción, mercado y empleo, orienta directamente el problema económico del empleo y el desempleo hacia las empresas de producción del conocimiento que son las Universidades y su funcionalidad. $Y$ ello conduce directamente a los profesionales de la producción del conocimiento y de la formación: los profesores. Si la Universidad es una institución formativa debe tener especialistas en formación: los profesores. $Y$ lógicamente deben pasar un periodo de formación inicial, un periodo de iniciación a la docencia y un periodo largo, permanente, de desarrollo profesional específico, que puede realizarse de forma colaborativa entre principiantes y mentores, o entre colegas analizando la propia la enseñanza en grupo, porque quien enseña a enseñar aprende a enseñar mejor.

Los profesores principiantes tienen especial necesidad de aprendizaje de la enseñanza y de apoyo. Mayor \& Sánchez (1998) concretan la posibilidad de apoyo a través de la figura del mentor en dos sentidos: en la dimensión de contenido como apoyo al aprendizaje de la tarea de enseñar, y como apoyo personal para la responsabilidad de la enseñanza como colega, o como parte integrante de un equipo. Lo cual es importante si ha de darse una coherencia a la formación. Dos tareas señalan como principalmente formativas, dentro de un marco de supervisión, que definen cómo trabajar con profesores para mejorar su enseñanza: la reflexión sobre las cuestiones de planificación, metodología, gestión de clase, motivación, aprendizaje etc. y la discusión sobre la forma de trabajar y de desarrollar la práctica y sus resultados.

Desarrollo profesional. Si la práctica docente no se analiza, se rutiniza. Si los conocimientos didácticos que se poseen no se cuestionan y no se aprende nada nuevo, la enseñanza se desprofesionaliza (Fernández Pérez, 1998). Si además, sabemos que los métodos que utilizan los profesores son los que se han aplicado en su enseñanza y en su aprendizaje, quiere decir que va a haber necesidad de aprender de otra manera, de entrar en otro tipo de aprendizajes a los ya conocidos, para poder empezar a cambiar la enseñanza. El desarrollo profesional y los programas de formación permanente son necesarios, e inevitables como programas de innovación para responder a los cambios en los que nos vemos inmersos sin buscarlos. Quizá los más generalizados son los cursos de duración breve o larga, los seminarios o grupos de trabajo permanente (de un a tres años); los grupos de innovación, en algunos casos son proyectos de innovación en la enseñanza que no implican a grupos de profesores sino a un solo profesor, que puede implicar a muy pocos alumnos; programas de intercambio entre países, como Intercampus etc.; $y$ por supuesto, la formación a distancia.

Pero la mejora de la Universidad no se conseguirá con la mejora de los profesores como individuos aislados, lo cual es importante, sino como miembros de una institución con una tarea valiosa que es la formación superior de los ciudadanos y profesionales de un país. 


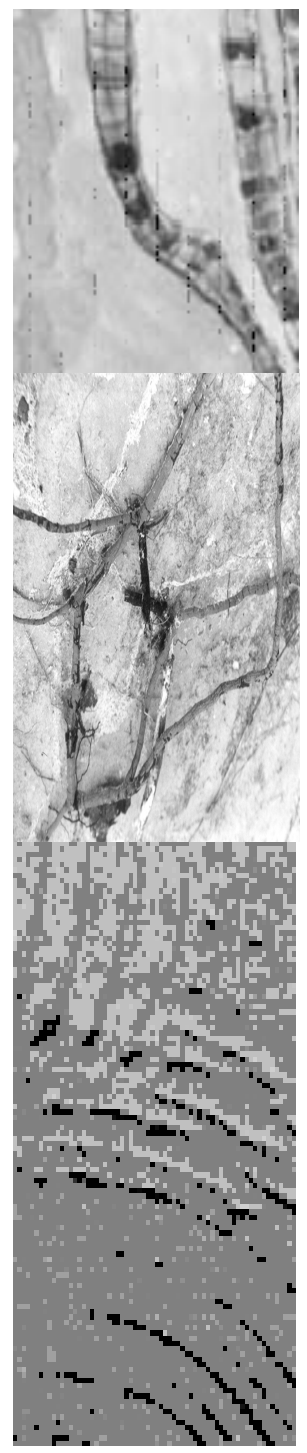

En todo caso la institución debe tomar en consideración este problema. La propuesta de formación de profesores universitarios de Benedito (1998) enfocada hacia el desarrollo profesional, abarca varios principios: establecer una política de formación y desarrollo universitario; crear un clima institucional favorable a la calidad y a la formación, una cultura de responsabilidad, colaboración y apoyo en el lugar de trabajo; considerando el Departamento como eje vertebrador de la Formación del profesorado, y dentro de él los grupos de trabajo e investigación como instrumentos de aprendizaje por la comunicación, la transferencia de información y de experiencia, y la cooperación en la búsqueda de propuestas, profundización en el análisis de la enseñanza y solución de problemas, como medios de formación por un modelo de aprendizaje situacional; potenciar el liderazgo académico y didáctico.

$Y$ tener un plan de mejora definido, que habrá que negociar con los participantes en un programa de formación para que aterrice en sus necesidades y problemas, aproveche sus recursos y potencie la energía de los profesores, de los equipos y de la institución. Un plan que puede orientarse por algunos principios claros y que debe proporcionar formación didáctica $y$ técnica, posibilitando la utilización de los recursos de las Nuevas Tecnologías.

Ello exige la formación de equipos voluntarios, sensibilizados con la necesidad de la formación, que sirvan como situación de formación, pero también como ejemplos y estímulo a otros grupos; y la creación de incentivos y de estructuras que lleven a los investigadores a trabajar en equipos interdisciplinares, en torno a proyectos temáticos, acabando con el aislamiento del trabajo científico. La UNESCO (1998a) recomienda establecer políticas de 'seguro de calidad'; es decir, acciones encaminadas a la motivación del personal, como otorgar a los docentes una condición económica y social conveniente, equiparable a sus iguales que trabajan en la industria.

Calidad de estudiantes. O sea, selección en función del mérito, teniendo en cuenta la capacidad y la motivación. La educación superior no es educación general, obligatoria. El problema actual es que los alumnos hacen pruebas de selectividad que otorgan a casi todos los que se presentan, un puesto escolar, pero en aquellos estudios en los que quedan plazas, al margen de que les interese y tengan capacidad. Un aspirante a enfermería (Diplomatura) puede entrar en Matemáticas (Licenciatura), por ejemplo, aunque haya suspendido matemáticas y haya aprobado en septiembre, por ello. $Y$ hay diferencias de nota de corte entre Universidades, hay también diferencia de sistemas de acceso; hace falta orientación de los estudiantes de Secundaria para que no ignoren posibilidades de estudio de carreras actuales, $y$ saturen otras más tradicionalmente valoradas; hace falta actuar sobre la demanda y sobre la oferta, coordinándose territorialmente, y facilitar la movilidad estudiantil con un sistema de becas ajustado a esa finalidad.

Calidad de infraestructuras. Las instituciones superiores deben prestar atención a las Nuevas Tecnologías de la Información y la Comunicación, a la Enseñanza a Distancia y de la Universidad Virtual. La 
inversión inicial es importante. Deben ser de buena calidad, y de última producción. O se queda obsoleta inmediatamente la formación que proporcionan.

Una cultura de rendición de cuentas, evaluación y regulación. Pero el trabajo a largo plazo de la Universidad, hace más difícil la evaluación de su impacto.

Evaluación como instrumento de calidad La evaluación es un requisito y una medida de las que primero se han adoptado. Evidentemente, para mejorar hace falta saber qué ocurre. Pero también por razones de interés de política internacional muy influenciada por el ámbito económico. En Europa la aspiración al mercado único de las profesiones obliga a la certificación de la formación y a la homologación de los títulos, que responda a la pregunta, por ejemplo, sobre ¿Qué sabe un ingeniero químico y qué sabe hacer? Lo haya cursado donde lo haya cursado. Ello tiene que ver con la evaluación de los alumnos. Sin embargo, en estos momentos, hay otros campos sometidos a evaluación en educación superior, como los profesores, la investigación y la gestión, como efecto de la propuesta de evaluación de la calidad total, derivada del modelo empresarial, y sobre los que haremos alguna reflexión.

\section{La evaluación del profesorado universitario}

La Evaluación en la Universidad ha comenzado siendo, en primer lugar, evaluación del profesorado (Art. 45.3 de la Ley de Reforma Universitaria), más tarde considerada como evaluación de la calidad de la docencia. De hecho, la evaluación de la calidad de la enseñanza en nuestros Estatutos está regulada por la Comisión de Docencia, un instrumento de control y de poder de los alumnos, que no se ha investigado hasta qué punto tiene relación con la evaluación de la calidad, y desde luego no sabemos qué relación tiene con la mejora de la calidad de la enseñanza. Aunque en algunos contextos la Comisión de Docencia del más alto nivel haya tomado alguna conciencia de la necesidad de unir la preocupación por la formación del profesorado junto a la de dar respuesta administrativa a las denuncias de los alumnos por cuestión de calificaciones.

Hay tres argumentos que avalan la evaluación de los profesores individualmente considerados: en primer lugar, el rendimiento de cuentas ya que el profesor está contratado para un servicio que debe ser útil y provechoso; en segundo lugar, la promoción, que debe tener en cuenta su competencia y su eficacia docente; y en tercer lugar, la mejora: la evaluación es un instrumento que debe proporcionar conocimiento sobre las fortalezas y las debilidades de un sistema, en este caso de enseñanza, del cual deben derivar las propuestas de cambio. De Miguel (1998), desde un enfoque más bien técnico, ha propuesto algunos criterios para la evaluación del profesorado: productividad; competencia docente; excelencia y desarrollo profesional.

La evaluación institucional de la Universidad En la actualidad, ha ido evolucionando el concepto de calidad y la

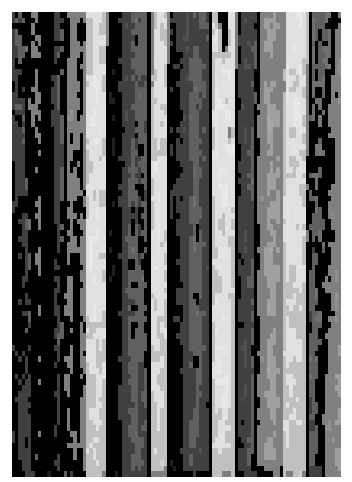


necesidad de evaluación de la calidad, percibiéndose la necesidad de que abarque a la institución. Por eso, evaluación institucional. ¿Cuáles son las razones? La misma autonomía. Un servicio público autónomo en una sociedad democrática, debe rendir cuentas a la sociedad que recibe sus servicios, y a la Administración que lo crea y lo financia. La Universidad debe rendir cuentas sobre qué hace, y cómo, y esto es un deber moral $e$ intelectual, no sólo legal (Rodríguez Espinar, 1998). Pero, además, hay otros argumentos: la expansión del sistema de enseñanza superior ha puesto de manifiesto sus debilidades; los altos costes son una presión hacia la eficiencia; la sociedad exige más aportaciones al desarrollo nacional; la internacionalización de la producción y de la formación superior reclaman niveles de calidad contrastados y contrastables; el derecho de los usuarios a conocer la calidad de la institución en la que se forma o desea formarse; "la naturaleza de las funciones y actividades de las instituciones universitarias reclama la existencia de procesos internos y externos de evaluación como procedimiento para garantizar la pertinencia, eficacia y eficiencia de las mismas" (Rodriguez Espinar, 1998, p.8). Desde un punto de vista didáctico la evaluación está íntimamente ligada a la calidad como a todo proceso de enseñanza. No se puede planificar sin evaluar, no se pueden hacer planes de mejora de la calidad sin evaluar ésta.

Un modelo de evaluación institucional, respecto a la metodología, creo que debe integrar la Evaluación Interna (Comité responsable de la evaluación interna), con la Evaluación Externa (Comité externo de evaluación), que desemboca en un Informe síntesis de ambos estudios, el cual debe ser sometido a una metaevaluación con el fin de valorar el propio informe, y a partir de ahí hacer las propuestas de mejora, que deberán ser seguidas por un plan de evaluación continua de las mejoras adoptadas. Por otra parte, dos criterios complementan la evaluación: Publicidad y participación (Plan Nacional de Evaluación de la Calidad de las Universidades).

La calidad de la investigación

La investigación es un motor de calidad. Pero como es una función universitaria, afecta a la calidad de la institución universitaria. Por ello, es preciso evaluar y mejorar la calidad de la investigación que produce y a su impacto. Previamente, es preciso poder investigar, y ello requiere recursos humanos, temporales y financieros. En la OCDE el promedio de gasto en investigación es del 2.2\% del PIB (en 1997), en España el 0,86\%. Pero de ello, el $52,4 \%$ en España correspondió a las Administraciones Públicas, cuando en la OCDE el promedio fue del 33,8\%

Supuesto todo ello, hay problemas que solucionar, porque la búsqueda de recursos y la justificación de los mismos no puede ser tarea de los que tienen que hacer la investigación, la calidad de la misma puede valorarse en relación con algunos criterios:

a) La respuesta a los problemas de la sociedad (los problemas de la sociedad son sus problemas de investigación. La calidad de la investigación exige la formación de investigadores, no sólo la evaluación. Este es un campo forzosamente de trabajo en equipo, de formación en equipo, y de 
colaboración con otros equipos; también interdisciplinar. Pero los problemas son teóricos y de la realidad, y hay que trabajar con los que viven en otras realidades que la universitaria).

Desde el punto de vista del trabajo, hay que pensar en la producción de conocimiento orientado a las necesidades de producción de las empresas. Ello supone estudiar las demandas y la prospección de demandas del mercado de trabajo, y organizar la investigación aplicada y la formación en esa línea de futuro; aunque no sólo eso. Los sistemas de educación superior deberían aumentar su capacidad para vivir en medio de la incertidumbre, para transformarse y provocar el cambio, para atender a las necesidades sociales y fomentar la solidaridad y la igualdad, preservar el rigor y la originalidad científicos con espíritu imparcial, como requisito para la calidad.

b) Los resultados de la investigación solucionan problemas, o se utilizan para buscar las soluciones oportunas. Es decir, qué nivel de impacto tiene la investigación en el conocimiento y en la realidad.

c) Se integra en las orientaciones y políticas de la Unión Europea y de la colaboración internacional, respecto a la búsqueda de la innovación en distintos campos de la actividad humana; también en la formación, y específicamente en todo lo relacionado con la necesidad de potenciar la cohesión social y el uso de las Tecnologías de la Información y la Comunicación, por su potencial para promover el aprendizaje a lo largo de toda la vida (Comisión de las Comunidades Europeas, 2000).

d) Supone la iniciativa en la investigación básica y la prospectiva en el planteamiento de nuevos problemas, nuevas formas de investigar, de definir y entender las realidades. La Universidad puede ofrecer la sabiduría del distanciamiento de los fenómenos y de las apariencias, su capacidad de previsión, y su interés por el largo plazo, su afán por la universalidad del conocimiento y la justicia para el desarrollo de un mundo más armonioso.

e) Se realiza en equipo integrando los saberes y técnicas de distintos profesionales y distintas áreas de conocimiento.

f) La evaluación externa por expertos debería enfocarse no sólo a efectos de reconocimiento curricular y económico, sino también a efectos de mejora. Ello supone información sobre criterios utilizados que pueden orientar los pasos siguientes en el trabajo de investigación.

Para finalizar, aprovechamos la recomendación de la UNESCO para una mejora de la Educación superior:

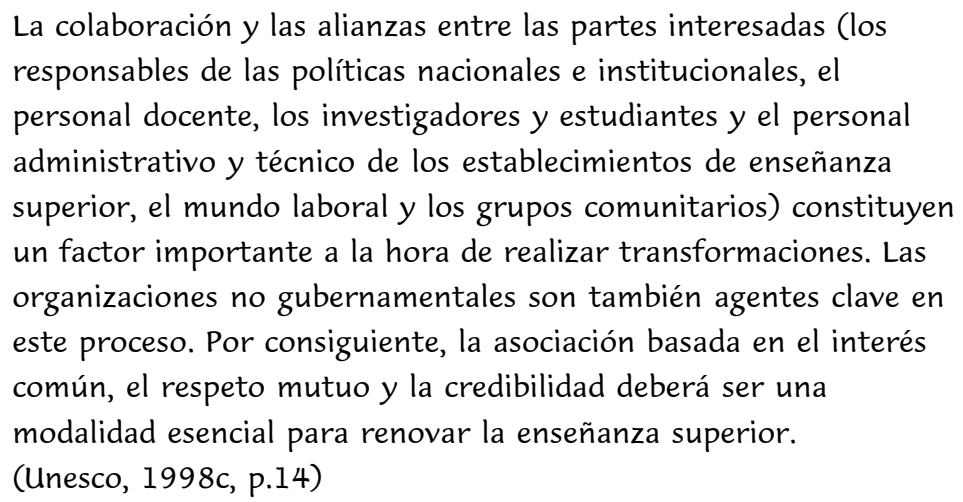




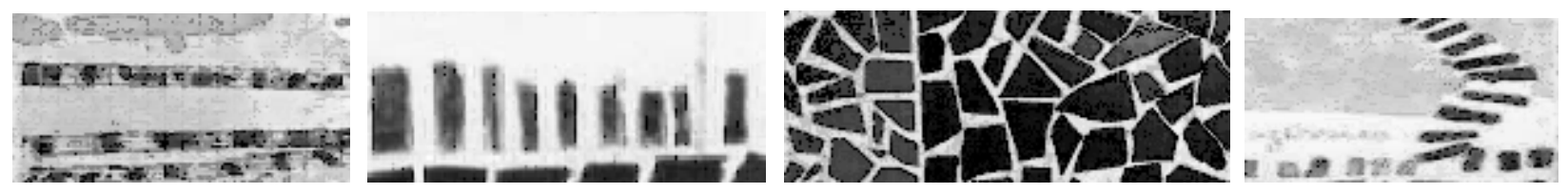

\section{Referencias}

BENEDITO, V. Hacia un modelo de formación inicial del profesorado universitario. In: DEPARTAMENTO DE EDUCACIÓN (Ed.). La formación del profesorado: evaluación y calidad. Las Palmas de Gran Canaria: Servicio de Publicaciones de la Universidad, 1998. p.51-72.

BRICALL, J. Informe Universidad 2000 para la Conferencia de Rectores de las Universidades españolas. Barcelona: CRUE, 2000.

COMISIÓN DE LAS COMUNIDADES EUROPEAS. Informe de la Comisión al Consejo y al Parlamento Europeo. Concebir la educación del futuro: promover la innovación con las nuevas tecnologías. Disponible en: <http://prometeus.org/eEurope/COM2000-23final/td.es.html>. Acceso em: 2 oct. 2000.

DE MIGUEL DIAZ, M. La evaluación del profesorado universitario. Criterios y propuestas para mejorar la función docente. Rev. Educ., n.315, p.63-83, 1998.

DELORS, J. La educación encierra un tesoro. Madrid: UNESCO, 1996.

DONDI, C. (Coord.) Proyecto OLD Policy Seminar. Política de apoyo de OLD en Europa. Bologna: Scienter, 1997.

FERNÁNDEZ PÉREZ, M. Formación del profesorado para el cambio. In: DEPARTAMENTO DE EDUCACIÓN (Ed.). La formación del profesorado: evaluación y calidad. Las Palmas de Gran Canaria: Servicio de Publicaciones de la Universidad, 1998. p.297-308.

GARDNER, H. La educación de la mente y el conocimiento de las disciplinas. Barcelona: Paidós, 2000.

HUBER, G. La Universidad del siglo XXI: la formación para la transformación social y la mejora del Empleo. In: MARTÍN-MORENO CERRILLO, Q.; MONCLÚS ESTELLA, A.; MEDINARIVILLA, A.; DOMÍNGUEZ FERNÁNDEZ, G. (Coords.) CONGRESO INTERUNIVERSITARIO DE ORGANIZACIÓNDE INSTITUCIONES EDUCATIVAS: LAS ORGANIZACIONES ANTE LOS RETOS EDUCATIVOS DELSIGLO XXI, 5., 1998, Madrid. Resumenes... Madrid: Departamento de Didáctica y Organización Escolar, 1998. p.89-97.

KYRIAKOU, D. Contexto económico de la formación: mercado de trabajo, política y modalidades de empleo. Sevilla: Grupo de Investigación IDEA!, 1997. Curso de experto en organización, desarrollo y evaluación de la formación profesional ocupacional. Módulo 5.

MARCELO, C.; ESTEBARANZ, A. Modelos de colaboración entre la universidad y las escuelas en la formación del profesorado. Rev. Educ., n.317, p.97-120, 1998.

MAYOR, C.; SÁNCHEZ, M. La implicación del profesorado universitario en su desarrollo profesional: la formación de equipos docentes en la universidad de Sevilla. In: DEPARTAMENTO DE EDUCACIÓN (Ed.). La formación del profesorado: evaluación y calidad. Las Palmas de Gran Canaria: Servicio de Publicaciones de la Universidad, 1998. p.533-46.

MICHAVILA, F.; CALVO, B. La universidad española hoy. Propuestas para una política universitaria. Madrid: Síntesis, 1998.

PÉREZ, R. Evaluación de programas educativos. In: MEDINA, A.; VILLAR, L. M. (Coords.). Evaluación de programas educativos, centros y profesores. Madrid: Universitas, 1995. p.73-131.

RODRÍGUEZ ESPINAR, S. La evaluación institucional en España. Análisis y planteamientos de futuro. In: 
GARCÍA, A. E.

LUXÁN, J.M. (Ed.). Política y reforma universitaria. Barcelona: CEDECS, 1998. p.131-58.

SÁENZ BARRIO, O. La universidad del siglo XXI. Un reto a la postmodernidad. In: MARTíN-MORENO CERRILLO, Q.; MONCLÚS ESTELLA, A.; MEDINA RIVILLA, A.; DOMÍNGUEZ FERNÁNDEZ, G. (Coords.) CONGRESO INTERUNIVERSITARIO DE ORGANIZACIÓN DE INSTITUCIONES EDUCATIVAS: LAS ORGANIZACIONES ANTE LOS RETOS EDUCATIVOS DEL SIGLO XXI, 5., 1998, Madrid. Resumenes... Madrid: Departamento de Didáctica y Organización Escolar, 1998, p.751-70.

TOFFLER, A. La tercera ola. Barcelona: Plaza y Janés, 1996.

UNESCO. Proyecto de Marco de Acción Prioritaria para el Cambio y el Desarrollo de la Educación Superior. In: CONFERENCIA MUNDIAL SOBRE LA EDUCACIÓN SUPERIOR, 1998, Paris. Documento de Trabajo... Paris, 1998a.

UNESCO. La educación superior en el siglo XXI. Visión y acción. In: CONFERENCIA MUNDIAL SOBRE LA EDUCACIÓN SUPERIOR, 1998, Paris. Documento de Trabajo... Paris, 1998b.

UNESCO. La educación superior en el siglo XXI. Visión y acción. Hacia un programa 21 para la educación superior. In: CONFERENCIA MUNDIAL SOBRE LA EDUCACIÓN SUPERIOR, 1998, Paris. Documento de Trabajo... Paris, 1998c.

ZABALZA, M. A. Los planes de estudio en la universidad. Algunas reflexiones para el cambio. Fuentes, n.1, p.27-68, 1999.

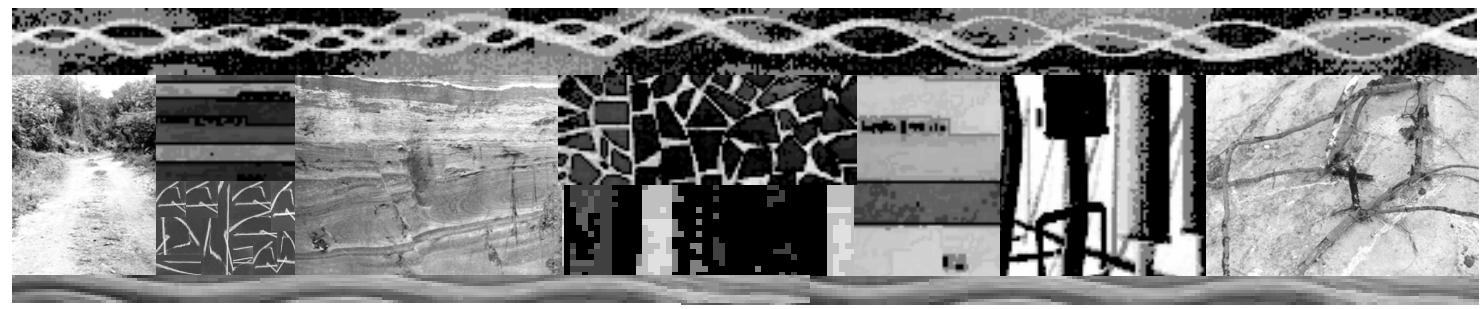

GARCÍA, A. E. Os caminhos da universidade em um contexto de mudança social, Interface - Comunic, Saúde, Educ, v.7, n.13, p.9-26, 2003.

Neste artigo faz-se uma reflexão sobre a problemática atual e os paradoxos da Educação Superior e apresentam-se perspectivas de aperfeiçoamento para um futuro imediato. Analisam-se as funções que a Educação Superior deve cumprir na sociedade atual, assim como as exigências de qualidade de uma formação que afeta um número cada vez maior de cidadãos, e da qual depende, em grande medida, a transformação social. Finalmente, são feitas propostas didático-pedagógicas para uma formação inovadora na Universidade.

PALAVRAS-CHAVE: Educação superior; mudança social; Pedagogia universitária. 